\title{
Application of Anorectal Manometry in the Treatment of Anorectal Diseases
}

\author{
Kai Wang ${ }^{1,2, a}$, Liping Liu' ${ }^{1,2, b}$, Yuyan Liu ${ }^{1,2, c}$ and Xiaoli Zhou ${ }^{3, d^{*}}$ \\ ${ }^{1}$ Henan Province Hospital of TCM, 450000, Henan, P. R. China \\ ${ }^{2}$ The Second Affiliated Hospital of Henan University of Traditional Chinese Medicine, 450000, Henan, \\ P. R. China \\ ${ }^{3}$ The Third Affiliated Hospital of Henan University of Traditional Chinese Medicine, 450000, Henan, \\ P. R. China \\ awk10236@126.com, bwkly1982@126.com, chnszyylyy@126.com \\ *dsfyzxl@126.com
}

*The corresponding author

Keywords: Anorectal dynamics; Packing colon disease; Clinical treatment

\begin{abstract}
Anorectal dynamics is a scientific study of the colon, rectum, anal canal (including pelvic floor) of a variety of motor function. Defecation occurs, and a variety of homemade anorectal diseases are associated with the development of colon, rectum, anal canal, related to changes in the mechanical state of the pelvic floor. Because of the many factors involved, complicated mechanism, as well as the limitations of detection methods, most physicians with only sketchy information on the patient complained of morphological and digital rectal examination, and X-ray provided to judge, but it is difficult for their function, especially functional movement state of qualitative and quantitative observation. In this paper, on this basis, the study focuses on the pathogenesis and treatment of colon disease packing method in clinical practice. For the latter to provide a theoretical basis for the treatment of colon disease packing.
\end{abstract}

\section{Introduction}

Padded stool colon disease refers to net long-term discharge is not retained in the intestines to the rectum or distal colon formed a solid mass of feces, but not by a relatively small segment of the anal canal is discharged via the rectosigmoid relaxation. Colon colon in the right iliac fossa continued in the cecum, in the third sacral plane connection rectum. Colon points ascending colon, transverse colon, descending colon and sigmoid colon 4, most fixed to the abdominal wall, the arrangement of the colon exactly like the English letter "M", enclosing the small intestine. Colon city includes the cecum, ascending colon, transverse colon, descending colon, sigmoid colon of five parts. As shown in Fig. 1.

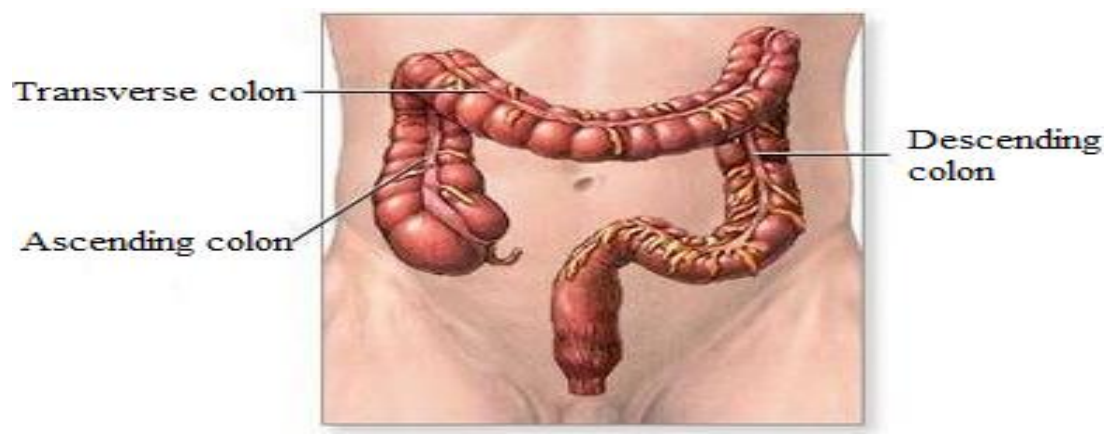

Figure 1. Colon schematic 
(1) Cecum: In the right iliac concave upper bound to the colon flap is bounded by the lower end of a dead-end. Cecum is starting end of the colon, the colon wall is thinnest, most superficial part of the location, about $6.25 \mathrm{~cm}$, width $7.5 \mathrm{~cm}$. Front and lateral cecum peritoneal covering, front and omentum and adjacent to the anterior abdominal wall, behind the iliac psoas muscle and adjacent pole position is not constant, it may be as high up under the liver or pelvic, and some mesangial long, very activities[1]. In the rear of the cecum and terminal ileum combined its top there is the inside of the appendix. In the ileum into the cecum wall at the entrance of the ileocecal valve, the ileocecal valve by the upper and lower lip folds components. Flap ends mesangial ileum and cecum sphincter ring formed by connecting these two circular muscle into the top and bottom, allowing the ileocecal valve has a sphincter, prevents colon contents reflux into the small intestine, but also control of food Mi will not be too quickly into the large intestine, small intestine so that food can be fully digested and absorbed.

(2) Ascending colon: By terminating the cecum, transverse colon is connected to the upper edge in the liver, long $12-20 \mathrm{~cm}$; and the front cover on both sides of the peritoneum, it is fixed to the abdominal wall and the pressure side wall; in front of the small intestine and large networks film and the anterior abdominal wall; the rear connected by loose connective tissue and abdominal wall, downward from the upper right kidney, back fascia, the inside of a more difficult descending duodenum, right ureter, surgical separation.

(3) Transverse colon: The colon is the longest and most active portion, long $40-50 \mathrm{~cm}$, in the ascending colon and hepatic flexure phase, in the splenic flexure and descending colon is connected, splenic flexure position generally higher than high hepatic flexure, transverse colon is the upper stomach, below is the small intestine, pancreas attached to the rear by the mesocolon, the front is covered by omentum. Transverse motion is large, sometimes down to the pelvis.

(4) The descending colon: From the transverse colon and splenic flexure connecting the upper and lower attached to the iliac crest level and sigmoid colon, length $20 \mathrm{~cm}$, in front and on both sides of the peritoneum covering the rear by loose connective tissue and the lateral left kidney, transverse abdominal aponeurosis starting waist side muscle contact. The shift behavior from the left flank and the outer edge of the left kidney along the waist down to the lower pole of left kidney, turning slightly to the inside of the side edge of the psoas muscle, and then between the psoas muscle and lumbar side down to the iliac bone crest level sigmoid colon.

(5) The sigmoid colon: In the pelvic cavity, located between the descending colon and rectum, on the shorter segment, called the iliac colon, lower long basin called the colon, the larger the difference in length for 20-70cm; intestine was "B" shaped bent named. Sigmoid mesangial more long, activity degree, sometimes volvulus can occur, followed by mesangial attached to the abdominal wall, followed by a downward opening between the sigmoid colon crypt. Colonoscopy should be in accordance with its shape, let it bend into the mirror.

\section{The Impact of Factors Anorectal Dynamics}

Anorectal dynamics in recent years developed a new discipline. Anorectal manometry through the viewing anorectal pressure, rectal sensation, anorectal reflex changes such as inhibition studies of normal anorectal physiology, physiological bowel control because of its relationship with anorectal dynamic changes and other reasons bowel dysfunction caused by the a discipline[2]. It is by statics and dynamics and EMG-based approach to the study of the colon, rectum, anal canal (including pelvic floor) of a variety of sport, and thus physiological defecation, anal homemade physiology and pathophysiology related anorectal diseases carry out one kind of research means research.

Related factors often affect anorectal dynamics include: psychological factors, changes in the pelvic floor muscles, pelvic floor nerve injury or disease, gastrointestinal hormone gastrointestinal salary membrane irritation, the concentration of No in the gastrointestinal tract, inflammatory mediators on Bo membrane irritation of the gastrointestinal tract, temperature and the like.

(1) Mental factors and psychological factors 
Psychological factors is one of the important factors that affect gastrointestinal function, recent studies have shown that psychological abnormalities have been associated with gastrointestinal functional disorders is an indisputable fact[3]. Constipation in patients with depression, anxiety, hypochondria and general psychological distress and other parties and are closely related, suggesting that psychological factors is one of the important factors affecting the anorectal dynamics. The role of psychological and social factors in anorectal disease more and more attention, it will be biological and psychosocial factors combined together for treatment of anorectal disease provide new ideas and methods. In recent years, there are many clinical studies have proved the existence of gastrointestinal functional disorders in patients with abnormal psychological factors, due to psychological factors, can cause brain function temporarily lose balance, autonomic dysfunction, peripheral nerve reflex hindered, anorectal disorders leaving the neural activity hormone secretion, gut a brain neurotransmitter system abnormalities, autonomic nervous excitability increased or decreased, resulting in contraction of the pelvic floor muscles dominated by abnormal, so that the gastrointestinal motility and visceral sensory function affected. Some scholars study confirmed that mood disorders or stress can alter the colon and small intestine movement, and can improve the sensitivity of the gastrointestinal tract visceral feeling, which plays an important role in functional gastrointestinal disease pathogenesis.

(2) Changes in the pelvic floor muscles

Pelvic floor is composed of various muscle groups to participate constituted, is closely related to changes of anorectal motility, and any diseased or damaged muscle groups will directly cause a change in anorectal dynamics. The main performance of the pelvic floor muscles during defecation or anal contraction abnormalities can not be completed effectively shrinking, anorectal manometry detection can be expressed as anal resting pressure, anal canal length function, defecation reflex anal pressure and other abnormal changes. Human pelvic floor is composed of various muscle groups to participate in the constitution. While closely related to changes in anorectal dynamics. Any muscle group of diseases and injuries will directly affect the anorectal power. Anal sphincter injury can cause incontinence. Incontinence anal sphincter damage caused by changes in anorectal dynamics inspection mainly anal resting pressure drop, high pressure zone to shorten the anal, rectal anal inhibitory reflex weakened mainly anal maximum systolic blood pressure can also have different degrees of reduced: incontinence and anal sphincter damage caused by anal places maximum systolic blood pressure decreased mainly anal resting pressure may also have different degrees of reduction, anal high pressure zone is shortened, anorectal inhibitory reflex weakened. Spastic pelvic floor syndrome, anorectal dynamics in the examination of the anal canal resting pressure and anal maximum systolic blood pressure was normal. But analog defecation, anal pressure not only to reduce, or even to abnormalities increased significantly. Puborectalis spasm syndrome, anorectal dynamics examination anal resting pressure and maximum systolic blood pressure higher than normal increases, anal high pressure zone extended; analog defecation anal pressure is normal or abnormally increased in. The patients with congenital megacolon, since the lower intestinal muscle membrane and Bo sympathetic ganglion cell dysplasia or absence of bowel can not make lesion relaxation, it was sustained contraction, peristalsis disappeared. The most typical dynamics is anorectal inhibitory reflex disappears, and the maximum tolerated rectal start feeling significantly increased capacity, increased rectal compliance, and maximum anal resting pressure and systolic blood pressure is normal.

Since the pelvic floor muscle innervation mainly from the genital plexus, belong to foreign nerves. It is generally believed that the pudendal nerve plexus refers to the portion of the lower energy or iliac plexus plexus plexus and tail between, mainly by skeletal nerve anterior branch (S2, S3, S4) constituted; regulation of the internal rectal nerve is mainly composed of the submucosa submucosal plexus and is located in the circular muscle, between the longitudinal muscle myenteric plexus[4]. In anorectal movement dominated, the first skeletal nerve is considered to be within the sphincter motor neurons, the nerve is considered anal sphincter motor neurons. So when anorectal plexus damage, rectum and anal canal expansion and intestinal feel illustrates the value may change (increase or 
decrease) occurs, it can cause initial rectal sensation, rectal maximum tolerable capacity change, rectal compliance change, and pelvic Floor achalasia syndrome of anal resting pressure and maximum systolic blood pressure is normal. Most patients with diabetic nerve damage, studies have found that patients with anorectal initial feeling of closure, defecation sensation Lang, defecation distress threshold and maximum tolerated closure were significantly higher than normal, which may be a diabetic patient autonomy neural and peripheral neuropathy.

(3) Changes in gastrointestinal hormones

Gastrointestinal hormone produced mainly by the gastrointestinal tract Qin film wherein MLT, SP (P substance), NT, GAS (gastrin) is an important excitatory neurotransmitter gastrointestinal motor neurons, V1P (vasoactive intestinal peptide), SS is an important inhibitory neurotransmitter gastrointestinal motor neurons, the movement through various forms of participation secrete digestive organ regulation.

(4) Changes of nitric oxide

Recent studies show that nitric oxide in the inner sphincter activity plays an important role. NO is a non-adrenergic, non-cholinergic neurotransmitter released from the gastrointestinal tract. Rattan found that NO can sphincter concentration-dependent relaxation response. Sphincter spasm in the Watson application of nitroglycerin therapy can reduce anal resting pressure of more than $25 \%$, due to an organic nitrate is nitroglycerin, which provides NO.

(5) Other factors

Other factors such as inflammatory mediators such as prostaglandins, nerve growth factor, bradykinin skin, 5-HT and adenosine and other stimuli, local inflammation, swelling, pain and irritation due to cable, meals, temperature, cough and other anorectal dynamics are have a certain impact.

\section{Clinical Application}

Studies have shown that is closely related to anorectal motility and paresthesia with functional constipation disease. Foreign scholars reported that patients with functional constipation feeling closed its initial value rectum and rectal defecation initial feeling closing values were significantly higher than the maximum tolerated capacity also tended to increase, which may account for rectal reduced sensitivity, increased tolerance, proved an important pathophysiological mechanisms of functional constipation patients feel abnormal formation of constipation. Cyclic incarcerated hemorrhoids, anal fissure, perianal abscess in patients with anal canal resting pressure, rectal resting pressure was evident, in a constant internal sphincter spasticity. Chronic anal fissure is due to anal skin ulceration, skin sensory nerve endings exposed to the outside, by repeated stimulation of inflammation and feces, can cause spasm of the internal anal sphincter, rectum and anal canal in a constant state of contraction. Perianal abscess is largely due to the internal anal sphincter, anal gland infection between the external sphincter, bacteria and infectious agents along the anal glands to the diffusion gap perianal abscess formation, since necrosis factor stimulate inflammation and tissue purulent, anorectal ring has been in a state of tonic contraction, severe localized pain, swelling. Sphincter spasm within the above categories of diseases of the anus or anorectal due to inflammation of various muscle stimulation in tight state, not as good as normal anal relaxation, so its anal, rectal resting pressure was significantly higher than normal, and anal canal diastolic blood pressure was lower than that of ordinary people. Ring mixed hemorrhoids, anal sputum of patients with anorectal pressure from ordinary people but no significant difference, just ring mixed hemorrhoids anal maximum systolic blood pressure is slightly lower than normal. Because all these muscle function in patients with anal sphincter, rectum muscle inner and outer longitudinal muscle and anorectal ring are in the normal state. If there is no secondary anal abscess phlegm, mixed hemorrhoids without thrombosis, the formation of local inflammation, but only in the anal cushion hemorrhoid venous plexus expansion vascular congestion, its impact on anorectal pressure is not great. Anal maximum systolic pressure is produced by the co-contraction of the pelvic floor muscles, the external sphincter, 
which is to maintain homemade anal function, especially under emergency conditions anal homemade main factors.

\section{References}

[1] Li Xue feng, Zou Yi you. Kinetics and psychological factors anorectal outlet obstruction constipation patients. World Journal of Gastroenterology, 200513 (1): 128-129.

[2] Xu Haishan, Jang You, Zhang Bolun. Studies in patients with chronic idiopathic constipation ship door rectum dynamics and psychological factors. Chinese Journal of Digestion, 2001 (6): 174

[3] Zhang Dong ge. Pelvic floor and anal Diseases [M]. Guizhou Science and Technology Press, 2000, 124-126.

[4] Li Shizhong. Colorectal anorectal dynamics change and its significance, Colorectal Surgery [J] 2002, 2 (8): 118-130. 